\title{
The conformational states of talin autoinhibition complex and its activation under forces
}

\author{
ZENG Yan ${ }^{1,3 \dagger}$, ZHANG Yong $^{1 \dagger}$, SONG XianQiang ${ }^{2,3}$, JI QingHua ${ }^{1,3}$, YE Sheng ${ }^{2}$, \\ ZHANG RongGuang ${ }^{2 *} \&$ LOU JiZhong ${ }^{1 *}$ \\ ${ }^{1}$ Key Laboratory of RNA Biology, Institute of Biophysics, Chinese Academy of Sciences, Beijing100101, China; \\ ${ }^{2}$ National Laboratory of Biomacromolecules, Institute of Biophysics, Chinese Academy of Sciences, Beijing100101, China; \\ ${ }^{3}$ University of Chinese Academy of Sciences, Beijing 100049, China
}

Received March 13, 2015; accepted April 2, 2015; published online May 29, 2015

\begin{abstract}
Talin is an integrin-binding protein located at focal adhesion site and serves as both an adapter and a force transmitter. Its integrin binding activity is regulated by the intramolecular autoinhibition interaction between its F3 and RS domains. Here, we used atomic force microscopy to measure the strength of talin autoinhibition complex. Our results suggest that the lifetime of talin autoinhibition complex shows weak catch bond behavior and does not change significantly at smaller forces, while it drops rapidly at larger forces $(>10 \mathrm{pN})$. Moreover, besides the complex conformation revealed by crystal structure, our molecular dynamics (MD) simulations indicate the possible existence of another stable conformation. Further analysis indicates that forces may regulate the equilibrium of the two stable binding states and result in the non-exponential force dependence of the binding lifetime. Our findings reveal a negative regulation mechanism on talin activation and provide a new point of view on the function of talin in focal adhesion.
\end{abstract}

cell adhesion, integrin signaling, single molecule biophysics, molecular dynamics simulations, conformational changes

Citation: Zeng Y, Zhang Y, Song XQ, Ji QH, Ye S, Zhang RG, Lou JZ. The conformational states of talin autoinhibition complex and its activation under forces. Sci China Life Sci, 2015, 58: 694-703, doi: 10.1007/s11427-015-4873-9

Talin is a largely abundant cytosolic protein containing more than 2500 amino acids. It links integrin and actin filaments, plays important roles in mechanical signaling pathway, and regulates various integrin-mediated cell adhesion events [1]. Structurally, talin can be divided into two parts: a globular N-terminal head and a large flexible C-terminal rod domain. Talin head includes a FERM domain (four-point-one-protein/ezrin/radixin/moesin) which contains three subdomain modules, F1, F2 and F3. The C-terminal rod domain is made of a series of $\alpha$-helical bundles and actin-binding motifs. Talin-FERM domain can bind with the cytoplasmic tail of integrin $\beta$ subunit via its

$†$ Contributed equally to this work

*Corresponding author (email: rzhang@ibp.ac.cn; jlou@ibp.ac.cn)
F3 subdomain. Talin head also contains the binding sites of various molecules such as PIPK $1 \gamma 90$ [2], F-actin [3], Ha-Ras [4] and phosphatidylinositol 4,5-bisphosphate ( $\left.\mathrm{PIP}_{2}\right)$ [5]. Talin rod binding proteins include vinculin [6,7], F-actin [8] and synemin [9]. Previous studies have indicated that talin not only forges the linkage between integrin and actin, but also serves as a regulator to active integrin [10-12] and as a force transducer between cell and extracellular matrix [13-15].

Talin head alone is sufficient for integrin activation, but full length talin is required for focal adhesions (FAs) assembly [10]. On the other hand, integrin activation is increased by expressing talin fragments with the head domain but not the rod domain [16]. That is, talin head has higher integrin-activating activity than full length talin, indicating 
that full length talin may exist as an inactive conformation [5]. Recent NMR and crystal structures showed that talin rod segment 1655-1822 (RS) could interact with talin-F3 $[17,18]$, which keeps talin inactive to prevent spontaneous integrin binding. The solved autoinhibition structures of talin F3/RS complex reveal that talin's activity may be regulated as well, although the molecular mechanism remains elusive.

Studies also find that Rap1 [19,20], RIAM [19,21], $\mathrm{PIP}_{2} / \mathrm{PIPKI} \gamma 90$ [5] and the interaction between talin and cell membrane [17] affect talin activation. Rap1 activates integrin by forming an integrin-associated complex containing talin and RIAM [20]. RIAM functions as scaffold that connects talin with Rap1, and the membrane targeting sequences in Rap1 recruit the complex to the membrane, thereby promoting talin binding to integrin [19]. Another talin regulator phosphatidylinositol 4,5-bisphosphate $\left(\mathrm{PIP}_{2}\right)$, a phospholipid component of plasma membrane, can bind with talin and block the interaction of talin F3/RS [5]. The $\mathrm{PIP}_{2}$-producting enzyme, type $\mathrm{I} \gamma$ phosphatidylinositol phosphate kinase (PIPKI $\gamma$ ), is also recruited during the process of talin activation [5], which results in the increase of $\mathrm{PIP}_{2}$ local concentration. PIPKI $\gamma$ and integrin $\beta$-tail share the same binding region in talin F3 domain [22], indicating that talin head must dissociate from PIPKI $\gamma$ before it can bind with integrin. The solved talin F2F3/RS autoinhibition structure reveals a negatively charged surface on talin-RS [17]. This feature, together with the fact that multiple positively charged residues locate at the proposed membrane binding surface of talin head, suggests a "pull-push" model of talin activation in which talin F2F3/RS interaction is broken by that inner membrane leaflet with accumulated anionic phospholipids strongly attracts positively charged surface of talin F2F3 domain and repels the negatively charged surface of talin RS domain [17].

Talin is not uniformly dispersed in the cell but locates dominantly at focal adhesion sites [23] where mechanical forces are generated between cell and matrix. As a force transducer, activated talin interacts with integrin on one side and cell skeleton actin filaments on the other side. It withstands the stretching force produced by various actin filament-associating molecular motors, the conformational changes of integrin and the charged inner leaflet of plasma membrane. It is obvious that forces may play significant roles for talin's function [24]. Not surprisingly, recent studies have indicated that force directly regulates focal adhesion dynamics [25-28]. As a molecule located at focal adhesion and a linker between integrin and actin filaments, activated talin must adapt the function at the complicated mechanical conditions in vivo.

At resting, talin exists in autoinhibition state which needs to be disrupted to release the talin head for integrin $\beta$-tail binding. This activation process of talin may be facilitated by mechanical forces as suggested by the "pull-push" mechanism. So far, little was known about the stability of talin autoinhibition and the dynamics of talin activation under force. To understand the potential effect of force and dynamic process on talin activation, we have investigated the dissociation of talin F2F3 and RS domain by single-molecule force spectroscopy using atomic force microscopy (AFM) and MD simulations. Our results suggest that talin F2F3/RS autoinhibition complex may interconverts between two stable conformations and the equilibrium can be regulated by applied forces. At smaller forces $(<10$ $\mathrm{pN}$ ), the lifetime of the complex does not have significant changes and may remain stable. When the force is larger than $10 \mathrm{pN}$, the lifetime of the complex decreases rapidly for fast disruption of the autoinhibition state. Our data provide further evidence for the "pull-push" model and are helpful to understanding how the inside-out signaling of integrin is accurately regulated.

\section{Materials and methods}

\subsection{Protein preparation}

Talin F2F3 (206-405) and RS (1654-1848) domains were prepared as previously described [5,17]. Briefly, the thrombin-cleavable N-terminal His-tag containing pET15b and pET30a vectors were used to clone talin F2F3 and RS, respectively. The vectors were expressed in BL21 E. coli and the resulting proteins were purified.

\subsection{AFM Setup}

A homemade atomic force microscopy (AFM), assembled using a well-established design [29], was used in this study. Talin F2F3 and RS domains were coated to the MLCT cantilever tips (Bruker Corporation, Arizona, USA) and the flat petri dish/glass surfaces respectively, and two different coating approaches were used. In the first approach, the proteins were linked covalently to the surface. The APTES (3-aminopropyltriethoxysilane) functionalized tips and glass surfaces were prepared according to the protocol in Ebner et al. [30]. The amino functionalized surfaces were then incubated with NHS-PEG3500-MAL (JenKem Technology Co., Ltd., Beijing, China) solution in PBS buffer at $4{ }^{\circ} \mathrm{C}$ overnight. After thoroughly washed with PBS, the tips and glass surfaces were incubated with F2F3 and RS respectively for 1 h. Both F2F3 and RS contain exposed cysteine residues on their surface (but none in their interaction interface, Figure S1 in Supporting Information) which can covalently link to MAL group on PEG tether. TBS buffer $\left(250 \mathrm{mmol} \mathrm{L}^{-1}\right.$ Tris, $150 \mathrm{mmol} \mathrm{L}^{-1} \mathrm{NaCl}$, pH 7.4) containing $1 \%$ Bovine serum albumin (BSA) was used as the working solution. In the second approach, MLCT cantilever tips and polystyrene petri-dish surfaces were directly incubated with F2F3 and RS protein solution respectively at $4^{\circ} \mathrm{C}$ overnight (Figure 1A). We used $1.4 \mathrm{mg} \mathrm{mL}^{-1} \mathrm{~F} 2 \mathrm{~F} 3$ and $0.7 \mathrm{mg} \mathrm{mL}^{-1} \mathrm{RS}$ in 

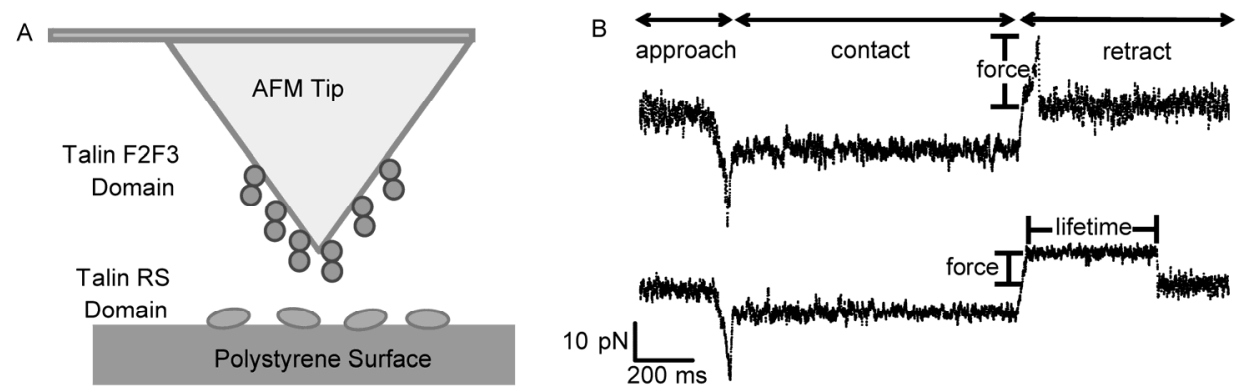

Figure 1 AFM experiments. A, The AFM functionalizing. AFM cantilever tip is coated with talin F2F3 domain and the polystyrene petri dish surface is coated with talin RS domain. B, Representative force traces of talin F2F3/RS dissociation for force-ramping (upper) and force-clamping (lower) experiments.

our experiments to achieve $\sim 20 \%$ successful adhesion events to ensure single molecule measurement. The similar setup was previously used to measure other protein-protein interactions [31-33] and the validity of the direct absorption method was confirmed. BSA was used as a substitute for talin RS in negative controls. We used the negative experiments and controlled the adhesion frequency to achieve a reliable specificity of our experiments. Negative experiments usually have a very low adhesion frequency $(<5 \%$, Figure S2 in Supporting Information). After washing six times with TBS buffer, the tips and dish surfaces were blocked with BSA solution (1\% BSA in TBS buffer) at room temperature for $1 \mathrm{~h}$ to block the uncovered surface.

In each experimental circle, the cantilever tip was first pressed on the surface for $1 \mathrm{~s}$ at force about $10 \mathrm{pN}$, and then lifted up at a given speed. The cantilever deflection was collected and analyzed to count the adhesion rates and to calculate the unbinding forces. In force-ramp experiment, the cantilever was continually lifted till the rupture of the molecular bond (Figure 1B, upper panel). In force-clamp experiment, when the pre-set force was reached, the piezo stopped pulling so that force was maintained by feed-back control until the molecular bond was ruptured. The practical force can be directly obtained from the force curve (Figure $1 \mathrm{~B}$, lower panel). It was found that the forces maintained at a certain value very well during the clamp. Thousands of adhesion events were collected for meaningful statistical analysis in each experiment.

\subsection{Measurement of rupture force}

Cantilever deflection signal change at bond rupture was used to calculate the rupture force. The loading rate was determined by linear fitting the linear region right before the unbinding event in the force versus time curves [34,35]. The histograms of the rupture forces were fitted with Gaussian distribution. The maximum force from the fitting was taken as the most probable unbinding force at certain loading rate. The relationship between peak force and loading rate can be described as [36]

$$
f^{*}=\frac{k_{\mathrm{B}} T}{x_{u}} \ln \left(\frac{r x_{u}}{k_{\mathrm{B}} T k^{0 F}}\right),
$$

where the peak force $\left(f^{*}\right)$ is proportional to the logarithm of the loading rate $(r) . k^{0 F}$ is the dissociation rate of proteins, $x_{u}$ is the distance from the native potential well to the energy barrier of transition state, $k_{\mathrm{B}}$ is the Boltzmann constant, and $T$ is the absolute temperature [36].

\subsection{Interaction lifetime processing}

The duration of individual bond lifetime was directly measured from force-clamp experiment. The lifetimes were binned by force. The mean lifetime at each force bin was obtained while the uncertainty was given by the standard error of the mean. The survival probability versus time for each force bin was calculated from lifetime data and fitted with multi-exponential decay model [37]:

$$
\left\{\begin{array}{l}
\ln (\text { Survival Frequency })=\ln \left[\sum_{i=1}^{n} \omega_{\mathrm{i}} \exp \left(-k_{\mathrm{i}} t\right)\right] \\
\text { Rupture Probability }=1-\sum_{i=1}^{n} \omega_{\mathrm{i}} \exp \left(-k_{\mathrm{i}} t\right)
\end{array}\right.
$$

where $t$ is time, $k_{i}$ and $\omega_{i}$ are the off-rate and fraction of the $i$ th state, respectively. The sum of the fractions equals 1 . One- $(n=1)$ and two-state $(n=2)$ models were tested. The off-rate has a relationship with force and can be defined by Bell equation [38]: $k_{i}=k_{i 0} \exp \left(a_{i} f\right)$, here $f$ is force, $k_{i 0}$ is the off-rate at zero force and $a_{i}$ is a parameter reactive compliance of the $i$ th state determined empirically by taking temperature and other unknown factors into account.

\subsection{Molecular dynamics simulations}

The initial structures of talin F2F3/RS complex were obtained from protein data bank (PDB code 4F7G). After addition of the missing hydrogen atoms, the protein was solvated in a rectangular water box $(\sim 8.7 \mathrm{~nm} \times 11.3 \mathrm{~nm} \times 11.8 \mathrm{~nm})$ with $\sim 116.4$ thousand atoms including $\sim 36.9$ thousand 
TIP3P water molecule models. Sodium and chlorine ions were then added to neutralize the system and maintain $\sim 0.15 \mathrm{~mol} \mathrm{~L}^{-1}$ ion concentration. After pre-equilibration of system in which protein backbone atoms were constrained, the subsequent production trajectory lasted $\sim 129 \mathrm{~ns}$ in NPT ensemble $(300 \mathrm{~K}, 1 \mathrm{~atm})$. The $\sim 64$ and $\sim 86 \mathrm{~ns}$ snapshots were chosen as initial structure for both constant velocity steered molecular dynamics (cv-SMD) and constant-force steered molecular dynamics (cf-SMD), in which the simulation boxes were enlarged to $(\sim 8.7 \mathrm{~nm} \times 19.1 \mathrm{~nm} \times 11.8 \mathrm{~nm})$ and $\sim 26.7$ thousand more TIP3P water molecule models were added to systems. In cv-SMD, the force constant was set to $\sim 7 \mathrm{pN} \AA^{-1}$ and the velocity was set to $5 \AA \mathrm{ns}^{-1}$. In cf-SMD, the constant force was set to $\sim 70 \mathrm{pN}$. During the simulation, Particle Mesh Ewald method was used to reduce the truncation error of electrostatic interactions. VDW interaction was computed using the switch method, in which the Lennard-Jones (LJ) 6-12 potential was calculated normally within $10 \AA$, and switched off smoothly between 10 and $12 \AA$. SHAKE method was used on all hydrogen-containing bonds to allow a 2 fs time step in the equilibration simulations. In all SMD simulations, the time step was set to 1 fs without rigid bond approximation used. All energy minimizations and MD simulations were carried out using the NAMD2.8 software package [39] and CHARMM22 force field for proteins with CMAP corrections [40]. System preparations, result analysis and illustrations were performed using the VMD program [41].

\section{Results}

\subsection{Validating surface functionalization methods with force-ramp experiments}

To characterize the talin F2F3/RS bonding activity and verify our coating methods, we first used both covalent and direct coating methods to determine the most probable rupture forces required to disrupt talin F2F3/RS binding with force-ramping measurements (Figure 1B, upper panel). The results confirm that $\mathrm{F} 2 \mathrm{~F} 3 / \mathrm{RS}$ has a medium bond strength compared with many other biomolecular interactions [42].

For the direct absorption coating, a total of 5,845 effective dissociation events were collected into histograms according to their loading rates ranging from 50 to $15,000 \mathrm{pN}$ $\mathrm{s}^{-1}$ (Figure 2A) and each force histogram contains at least 200 unbinding events. These histograms all exhibited single force peak and the peak shifted toward larger forces with the increasing loading rate (Figure 2A). We fitted the force histograms with Gaussian distributions to analyze the dissociation kinetic with dynamical force spectroscopy (DFS) $[36,43,44]$. The peak force was plotted against the loading rate in a log scale, and the resulted loading rate dependent rupture force displayed two linear segments (Figure 2B).

When F2F3 and RS covalently attached on the tip and coverslip with the NHS-PEG-MAL linker, the successful
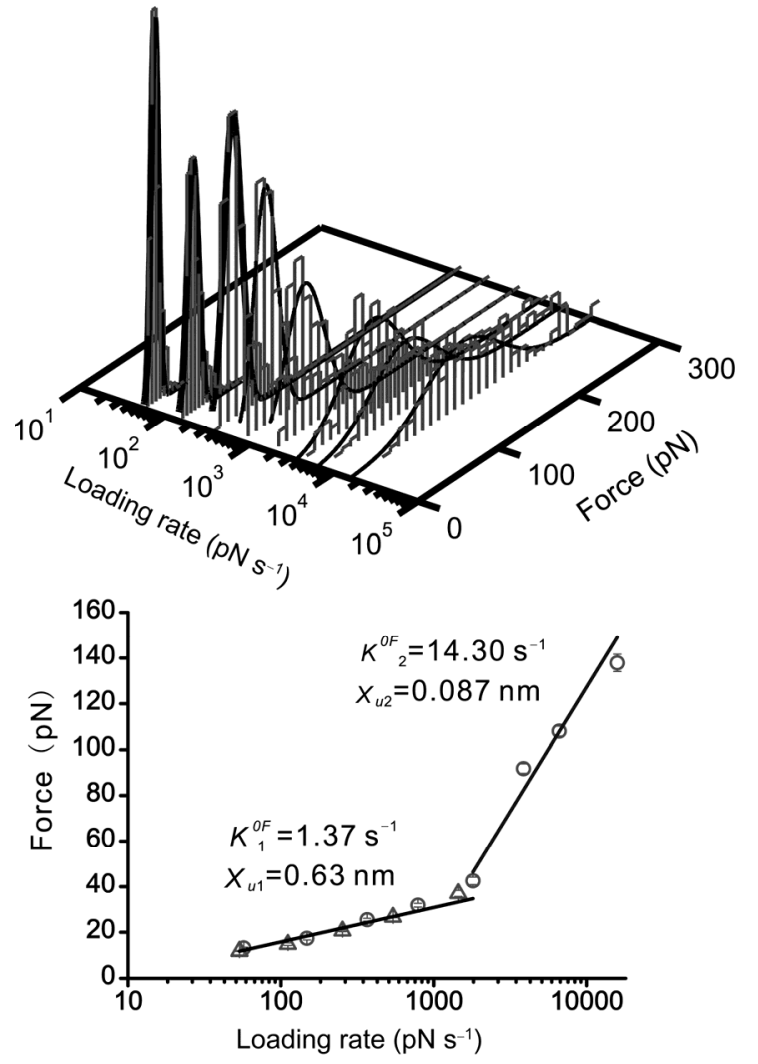

Figure 2 Dynamical force spectroscopy of talin F2F3/RS interactions. A, The rupture force histograms of unbinding forces of talin F2F3/RS dissociation at different loading rates. Each force histogram shows single force peak and is fitted with Gaussian distribution (solid lines). The most probable unbinding forces are determined from the fitting. B, The most probable unbinding forces are plotted against the loading rate in the log scale from direct adsorption experiment setup (circle) and covalently adsorption with PEG linker setup (triangle). The black and gray lines are fitting results using DFS theory. Fitting parameters are indicated.

rate in our experiment reduced dramatically and we can only collect enough data for the DFS analysis at the lower loading rate regime. Nevertheless, the covalent coating generated similar results in DFS as the direct absorption coating (Figure 2B), which indicates that the direct absorption coating is acceptable to study talin F2F3/RS interaction. We only use this method in our experiments to measure the force-dependence lifetime of talin F2F3/RS autoinhibition complex.

\subsection{Force-dependent lifetime of talin F2F3/RS bond}

Measuring force-dependent bond lifetime can provide more information than DFS analysis. We measured the lifetimes of talin F2F3/RS bond under various constant forces by force-clamp experiments. In the force-clamp experiments, when a pre-set force is reached, AFM controlling program maintains that force via force feed-back loop till the dissociation of talin F2F3/RS bond, as shown in Figure 1B lower panel. For each successful lifetime measurement, a lifetime and the corresponding force were obtained. A total of 1,239 
lifetimes were collected and then binned into six force groups. The average forces and average lifetimes in the six groups were used to plot the force-lifetime curve as shown in Figure 3A. Interestingly, as force increases from 5 to 10 $\mathrm{pN}$, the average lifetimes are slightly increased, which display weak catch bond behavior. After reaching its maxima at $\sim 10 \mathrm{pN}$, the average lifetimes decrease rapidly with further increase of force, showing the intuitive slip bond behavior.

Catch bond, whose lifetime is prolonged by applied force, and catch-slip transition, have been observed in various receptor/ligand interactions [29,37,45-47], actin-actin [48] and actin-myosin [49] interactions. To our knowledge, this is the first time to observe catch bond-like interaction in the autoinhibition interactions. It is noted that the catch bond behavior we observed (Figure 3A) is weak. The difference of average lifetimes at $\sim 5 \mathrm{pN}$ and that at $\sim 10 \mathrm{pN}$ is not statistically significant. Therefore, it is safe to say that from 5 to $10 \mathrm{pN}$, the average lifetime does not change too much.

The survival probability of talin F2F3/RS interaction for each force bin is shown in Figure 3B. It is found that the survival probability does not decay exponentially. Similar feature has also been observed in integrin/ligand interactions $[29,37,46]$. Multi-exponentially distributed lifetimes usually indicate that the dissociation may come from multiple populations of binding states. This suggests that the talin F2F3/RS interaction may form multiple stable conformations.

\subsection{MD simulations revealed two conformations in talin F2F3/RS binding}

To gain insight on the structural mechanism of talin autoinhibition interaction, we carried out the conventional MD and SMD simulations to study the dynamics and forceinduced dissociation of talin F2F3/RS complex. A clustering analysis was performed to the equilibration trajectory
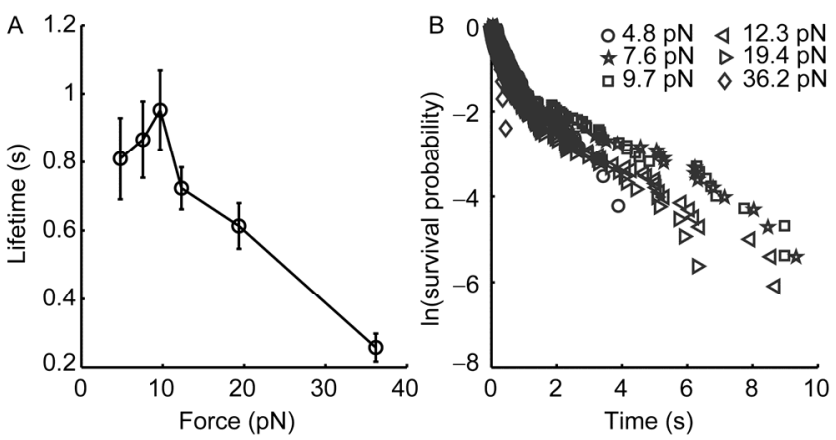

Figure 3 Force dependent lifetime in force-clamp experiments. A, Bond lifetimes of talin F2F3/RS interaction show catch bond-like behavior at smaller force regime and catch-slip transition. Each point represents mean lifetime \pm SEM of $>60$ measurements, except the last point at $36.2 \mathrm{pN}$ (in which 11 events were observed). B, The lifetime distribution (plotted as survival frequency) shows multiple exponential decaying feature. Each force corresponds to one data point in (A). using K-means clustering algorithm with a cutoff value of $8 \AA$ [50] and the results show the existence of two conformational clusters (Figure 4A, left panel). In the two clusters, which correspond to two binding modes, the relative position of F3 and RS domain is different and the $\sim 64$ ns (binding Mode 1) and $\sim 86 \mathrm{~ns}$ (binding Mode 2) snapshots can be used as the representative conformation for each binding mode. We classified simulated structures into different modes by its RMSD to the represented conformation in each mode ( $4 \AA$ is used as a cutoff). RMSD of RS domain after aligning F3 domain suggests that $\sim 20 \%$ simulated conformations are close to Mode 1 and $\sim 80 \%$ conformations are close to Mode 2 (Figure 4A, right panel). The two binding modes show structural similarity and differences. The hydrogen bond network observed in both conformations is shown in Figure $4 \mathrm{~B}$ and the differences are indicated in Figure 4C and D. Hydrogen bonds of K320-Q1774, K332-E1805 and Y377-D1676 are not presented in Mode 2 (Figure 4C and D).

Compared with the crystal structure of talin autoinhibition complex, the overall RMSDs of the two conformations based on $\mathrm{MD}$ are $\sim 2.0$ and $4.5 \AA$ respectively. And the RMSDs for the RS domain is $\sim 2.8$ and $6.9 \AA$ when the F2F3 domain is aligned. Mode 1 is closer to the crystal structure. To study the difference of interaction patterns among those structures, a superimposition of the RS domains in those structures was carried out. Interestingly, the F2F3 subdomains in Mode 1 and Mode 2 undergo significant rotations (about $14^{\circ}$ and $24^{\circ}$ for Mode 1 and 2, respectively) with one of the two protruding fingers of F3 (K316-P327) as axis, leading to increased distances between those two fingers (about 2 and $5 \AA$ more for Mode 1 and 2, respectively) (Figure S3 in Supporting Information). As a result of the rotation in Mode 2, the $\beta$-turn (S362-S365) close to the $\mathrm{N}$-terminus of the C-terminal helix of F3 approaches the RS domain, forming a hydrogen bond between S362 and H1755, a new connection that does not exist neither in the complex crystal structure nor in Mode 1 (Figure 4D).

The snapshots at 64 and $86 \mathrm{~ns}$ were then taken as the representative conformations for the two binding modes respectively for further SMD simulations. Two series of cv-SMD simulations (Figure 4E) were carried out using both snapshots as the initial structure, in which four different pulling strategies were applied (Figure 4F). The time-force curves show that the force peaks in Mode 1 cv-SMD simulations are larger than those in Mode 2 cv-SMD simulations for all pulling strategies, which suggests that the binding in Mode 1 is stronger than that in Mode 2. When a constant force $(\sim 70 \mathrm{pN})$ is applied to $64 \mathrm{~ns}$ snapshot conformation (Mode 1), the conformation of the complex is stable and does not have any significant change in $40 \mathrm{~ns}$ (Figure 4G). However, if the same constant force is applied to $86 \mathrm{~ns}$ snapshot conformation (Mode 2), a conformational change to Mode1 was observed at $~ 10 \mathrm{~ns}$, and lasted till the end of the simulation (Figure $4 \mathrm{H}$ ). These re- 

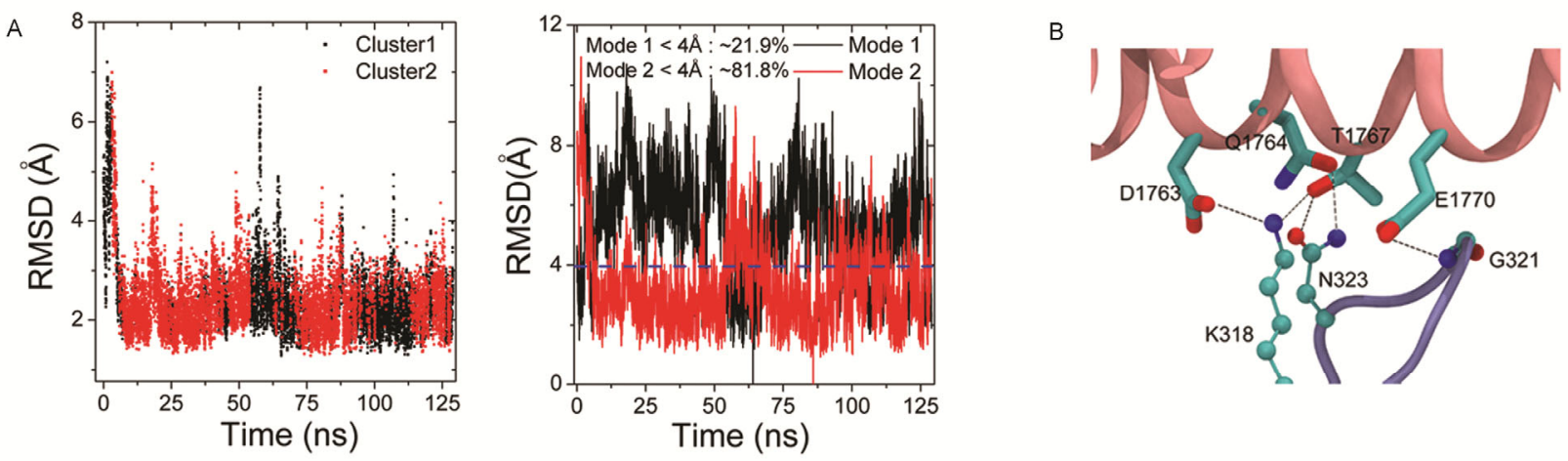

C

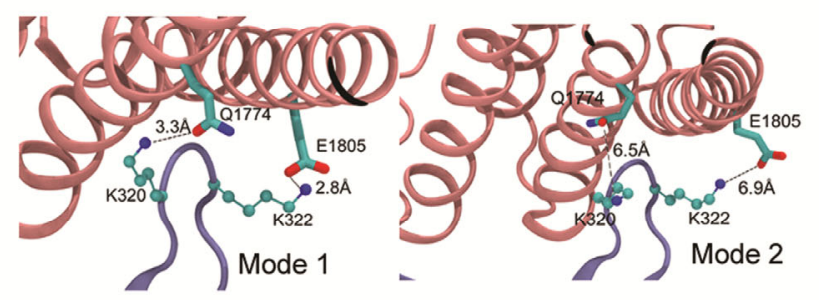

D
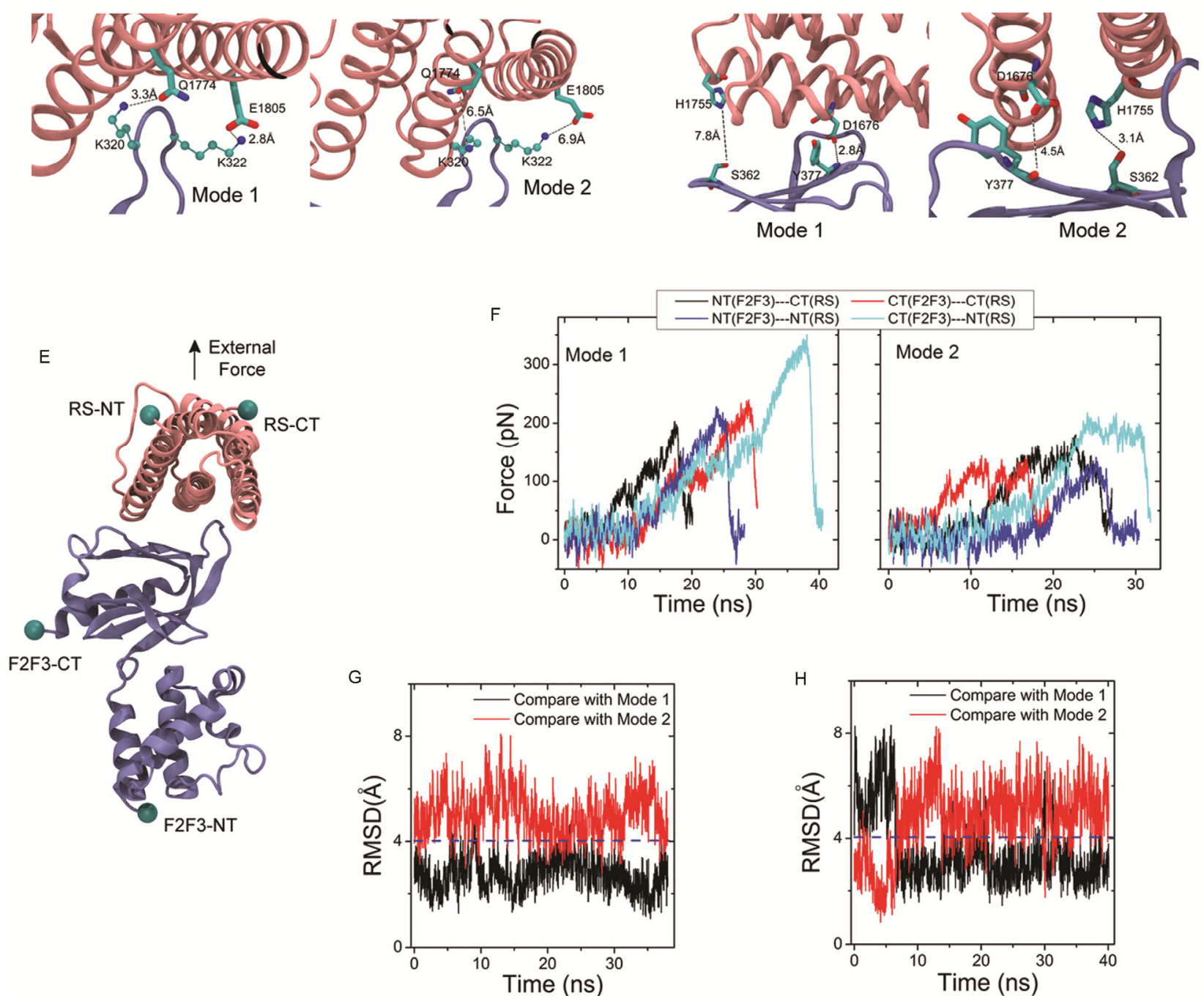

Figure 4 MD simulations of F2F3/RS complex. A, Left panel: conformational clustering for the 129 ns equilibration period using K-means clustering algorithm. Two clusters are obtained (black and red square). Right panel: RMSD of RS domain in the equilibration period relative to two selected conformations ( 64 and $~ 86$ ns snapshot, termed as Mode 1 and Mode 2), which are from cluster 1 and 2 respectively. B, Hydrogen bond network observed in both binding modes. C, Additional hydrogen bonds observed in binding Mode 1. D, Additional hydrogen bonds observed in binding Mode 2. E, The pulling strategies in SMD simulations. Talin F2F3 domain is harmonically constrained (N- or C-terminal) and the force is applied on the RS domain (N- or C-terminal). F, The time versus force curves of cv-SMD for the disassociation of talin F2F3/RS complex. The simulations of Mode 1 result in larger forces than those of Mode 2 for all pulling strategies. NT(F2F3)-CT(RS) indicates that N-terminal C $\alpha$ atoms of F2F3 are constrained and C-terminal C $\alpha$ atoms of RS are pulled, other strategies are named with the same rules. G and H, RMSDs of the cf-SMD trajectories with the representative Mode 1 and Mode 2 conformations. The simulation is from Mode 1 conformation $(\mathrm{G})$ or Mode 2 conformation $(\mathrm{H})$. It is clearly shown that Mode 1 is stable under the force but Mode 2 undergoes the conformation change to Mode 1 quickly after the force application. 
sults suggest that the complex maintains an equilibrium between the two binding modes at force free condition and the binding Mode 2 is favored. Force can shift the equilibrium and favor the binding Mode 1.

\subsection{Characterizing talin autoinhibition states and their regulations by force}

Since MD simulations indicate that two possible stable binding states exist for talin autoinhibition complex, it is straightforward to propose that the unusual force-dependent kinetics revealed in single molecule experiments result from the mixing of these two states whose fractions are shifted by the applied force. Similar properties have been observed in LFA-1 integrin/ICAM-1 binding previously [37,46]. To get further information on how force may regulate the fractions of the two binding states, we analyze the survival frequency data (Figure 3B) with a two-state model. In the model, each of the two states follows the first-order dissociation kinetics with the dissociation rate described by Bell model [38]. Our analysis indicates that the two-state model can fit the lifetime distributions of all forces very well (Figure S4 and Table S1 in Supporting Information). The resulting dissociation rates and the corresponding fractions are shown in Figure 5. At the weak catch bond regime $(<10 \mathrm{pN})$, the fractions of the two states do not have significant change, and the lower affinity state (larger dissociation rate, shorter lifetime, circles in Figure 5) is favored (Figure 5B). After the force increases to the slip bond regime $(>10 \mathrm{pN})$, the fraction of the lower affinity state deceases and that of the higher affinity state (smaller dissociation rate, longer lifetime, diamond in Figure 5) increases. At $36.2 \mathrm{pN}$, the fraction of the higher affinity state approaches $100 \%$ and that of the lower affinity state almost goes down to 0 .

It could be observed from the curve that the population of lower affinity state is $\sim 80 \%$ and the higher affinity sate is $\sim 20 \%$ at low forces (Figure 5B). This is in agreement with the fraction of the two states observed in MD simulations without the force application. These results indicate that two states obtained from analyzing the experimental lifetime data may correspond to the two binding states observed in MD simulations.
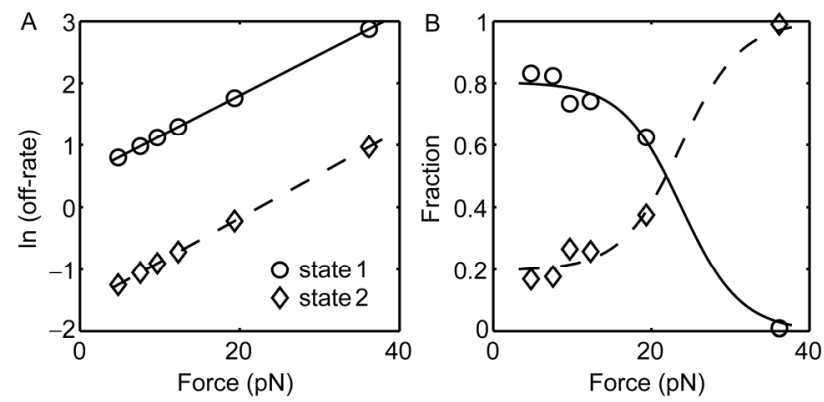

Figure 5 Analysis of lifetimes by two-state model. The off-rate (A) and fractions of each state (B) are plotted against the applied force.

\section{Discussion}

The autoinhibition interaction of talin through its F2F3 and RS domains is critical for integrin activation, which is responsible for the formation of focal adhesion. The recently solved crystal structure of talin F2F3/RS complex provides information at atomistic detail on the mechanism of the autoinhibition, and a "pull-push" model for talin activation is proposed based on this structure [17].

Our AFM measurements provided the force-dependent lifetime of talin autoinhibition complex. At smaller forces, the lifetime does not change significantly with the applied force, and only after the force increases above $10 \mathrm{pN}$, the lifetime of the complex begins to drop rapidly.

Our MD simulation indicated that talin autoinhibition complex may adopt another conformation besides the conformation solved in the crystal structure. The equilibrium between the two conformations are regulated by the mechanical forces, which result in the catch-slip transitional force dependence in the dissociate kinetics. It should be noted that although the MD simulations suggest the possible structural basis for the catch bond behavior we observed in AFM experiments, the physiological relevance of those conformations is not clear currently and needed to be justified. Combining NMR experiment and HADOCK modeling, an autoinhibition complex conformation is previously reported [18] which is different from the crystal structure [17]. Detailed comparisons show both the stable conformations observed in MD are not the same as the NMR one. We had also performed simulations starting from the NMR structure [18], a tendency of changing toward the crystal structure is observed (data not shown).

The single molecule biophysics and MD simulation studies provide further evidences for the "pull-push" mechanism. In the "pull-push" mechanism, talin is autoinhibited via the binding of its head F2F3 domain with the tail RS domain, its activation results from the attraction between the anionic lipids in plasma membrane and the positively charged residues in F2F3 domain (the "pull"), and the repulsion between the anionic lipid and the negatively charged residues in the RS domain (the "push"). Our studies are consistent with the pull-push mechanism and provide new evidence on how the activation is accurately controlled. A more detailed "pull-push" hypothesis is illustrated in Figure 6. Without any stimulus, the negatively charged phosphoserine (PS) is randomly distributed in the inner leaflet of the plasma membrane, and the phosphoinositol (PI) lipid is not phosphorylated. The autoinhibited inactive talin may attach and detach with the membrane frequently, but both the "pull" and the "push" forces are not strong enough to break the autoinhibition interaction (Figure 6A). If the PI lipid is phosphorylated non-specifically, the "pull" and "push" force will increase, because the lifetime of talin F2F3/RS binding does not change much at moderate force 

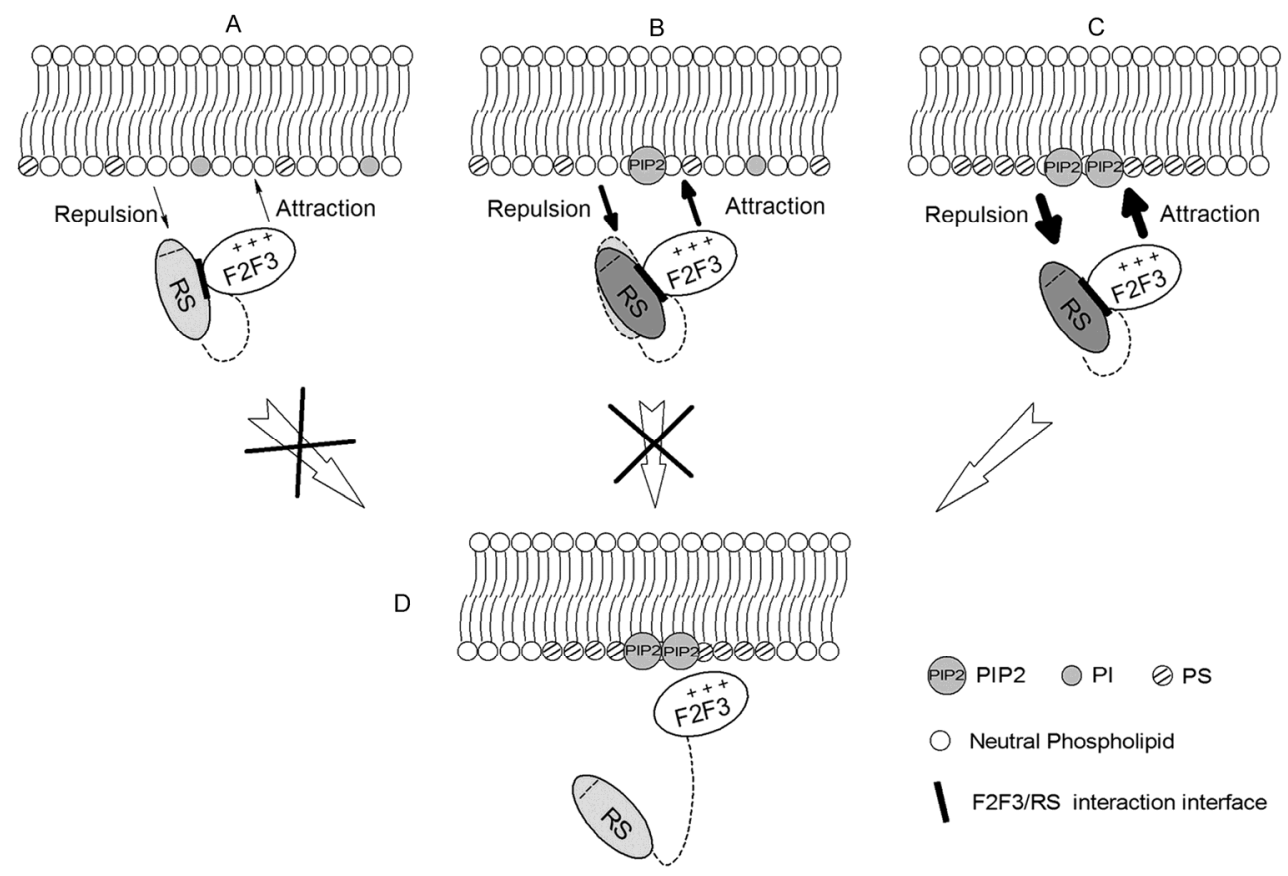

Figure 6 The precisely controlled "pull-push" mechanism of talin activation. Randomly distributed negatively charged PS lipid (A) or non-specifically phosphorylated PI lipid (B) cannot activate talin. Talin autoinhibition complex can only be disrupted in fully activated cell when PS and specifically phosphorylated PI lipids are accumulated locally in lipid membrane $(\mathrm{C})$. Activated talin binds with integrin at the FERM end and actin filaments at the rod end to form the mechanotransduction pathway (D).

$(<10 \mathrm{pN})$, these occasional PI phosphorylation still cannot activate talin (Figure 6B). Only after the cell is stimulated, $\mathrm{PIP}_{2}$ is systematically phosphorylated by PIPKI $\gamma$ kinase and PS phospholipids are also enriched locally, the "pull" and "push" forces exceed the threshold, talin autoinhibition complex can be dissociated and talin is activated (Figure $6 \mathrm{C})$. The activated talin then binds with integrin $\beta$ tail via the F3 domain and the talin rod domain will bind with actin filaments to connect the mechanotransduction pathway (Figure 6D). Thus, through the fine-tuned dissociation kinetics of the autoinhibition complex, talin's functional roles in focal adhesion may be precisely regulated. Briefly, talin activation is not a simple outcome of its membrane association. Only after the accumulation of negatively charged lipids (PS and/or phosphorylated PI) exceeds certain threshold, can attraction forces for the talin head and repulsion forces for the talin tail be strong enough to break F2F3/RS complex and activate talin. The catch bond behavior of talin F2F3/RS interaction may ensure that talin can only be activated when the activation is required.

As mentioned in the Introduction, many other factors may participate in talin activation process. For example, recently, RIAM was found to be able to block the RS-binding site on F3 and trigger talin activation [21], which suggests an alternative mechanism other than the "pull-push" model. We would like to emphasize that the two models do not contradict with each other. Talin activation is a multi-step process in the complex cell environment, and multiple mechanism and factors may co-exist and syn- ergistically accomplish this process. The active and inactive states of talin are in dynamical equilibration regulated by many biochemical and biophysical aspects, and various factors may influence its active or inactive rate. Here we mainly discuss that force regulation of the dissociation rate, other factors which could block autoinhibition binding sites can reduce the inactive process.

In conclusion, we found a negative regulation threshold for the "pull-push" mechanism of talin activation, and our results are important to understanding the precise regulation in talin's activation.

The computational resources for the MD simulations were provided by the Supercomputing Center of Chinese Academy of Sciences and National Supercomputing Center Tianjin Center. We thank Dr. Kong Fang and Dr. Lin JiangGuo for assistants in AFM design and experiment techniques. This work was supported by the National Basic Research Program of China (2014CB910202) and National Natural Science Foundation of China (11302240, 31070827 and 31222022).

1 Critchley DR. Biochemical and structural properties of the integrin-associated cytoskeletal protein talin. Annu Rev Biophys, 2009, 38: 235-254

2 Di Paolo G, Pellegrini L, Letinic K, Cestra G, Zoncu R, Voronov S, Chang SH, Guo J, Wenk MR, De Camilli P. Recruitment and regulation of phosphatidylinositol phosphate kinase type 1 gamma by the ferm domain of talin. Nature, 2002, 420: 85-89

3 Lee HS, Bellin RM, Walker DL, Patel B, Powers P, Liu HJ, Garcia-Alvarez B, de Pereda JM, Liddington RC, Volkmann N, Hanein D, Critchley DR, Robson RM. Characterization of an actin-binding site within the talin ferm domain. J Mol Biol, 2004, 343: 
$771-784$

4 Goldfinger LE, Ptak C, Jeffery ED, Shabanowitz J, Han JW, Haling JR, Sherman NE, Fox JW, Hunt DF, Ginsberg MH. An experimentally derived database of candidate ras-interacting proteins. J Proteome Res, 2007, 6: 1806-1811

5 Goksoy E, Ma YQ, Wang XX, Kong XM, Perera D, Plow EF, Qin J. Structural basis for the autoinhibition of talin in regulating integrin activation. Mol Cell, 2008, 31: 124-133

6 Burridge K, Mangeat P. An interaction between vinculin and talin. Nature, 1984, 308: 744-746

7 Gilmore AP, Burridge K. Regulation of vinculin binding to talin and actin by phosphatidyl-inositol-4-5-bisphosphate. Nature, 1996, 381: 531-535

8 Calderwood DA, Ginsberg MH. Talin forges the links between integrins and actin. Nat Cell Biol, 2003, 5: 694-697

9 Sun N, Critchley DR, Paulin D, Li ZL, Robson RM. Identification of a repeated domain within mammalian alpha-synemin that interacts directly with talin. Exp Cell Res, 2008, 314: 1839-1849

10 Zhang X, Jiang G, Cai Y, Monkley SJ, Critchley DR, Sheetz MP. Talin depletion reveals independence of initial cell spreading from integrin activation and traction. Nat Cell Biol, 2008, 10: 1062-1068

11 Wegener KL, Partridge AW, Han J, Pickford AR, Liddington RC, Ginsberg MH, Campbell ID. Structural basis of integrin activation by talin. Cell, 2007, 128: 171-182

12 Tadokoro S, Shattil SJ, Eto K, Tai V, Liddington RC, de Pereda JM, Ginsberg MH, Calderwood DA. Talin binding to integrin beta tails: a final common step in integrin activation. Science, 2003, 302: 103-106

13 Giannone G, Sheetz MP. Substrate rigidity and force define form through tyrosine phosphatase and kinase pathways. Trends Cell Biol, 2006, 16: 213-223

14 Jiang GY, Giannone G, Critchley DR, Fukumoto E, Sheetz MP. Two-piconewton slip bond between fibronectin and the cytoskeleton depends on talin. Nature, 2003, 424: 334-337

15 Giannone G, Jiang G, Sutton DH, Critchley DR, Sheetz MP. Talin1 is critical for force-dependent reinforcement of initial integrincytoskeleton bonds but not tyrosine kinase activation. J Cell Biol, 2003, 163: 409-419

16 Calderwood DA, Zent R, Grant R, Rees DJG, Hynes RO, Ginsberg MH. The talin head domain binds to integrin beta subunit cytoplasmic tails and regulates integrin activation. J Biol Chem, 1999, 274: 28071-28074

17 Song XQ, Yang J, Hirbawi J, Ye S, Perera HD, Goksoy E, Dwivedi P, Plow EF, Zhang RG, Qin J. A novel membrane-dependent on/off switch mechanism of talin ferm domain at sites of cell adhesion. Cell Res, 2012, 22: 1533-1545

18 Goult BT, Bate N, Anthis NJ, Wegener KL, Gingras AR, Patel B, Barsukov IL, Campbell ID, Roberts GCK, Critchley DR. The structure of an interdomain complex that regulates talin activity. J Biol Chem, 2009, 284: 15097-15106

19 Lee HS, Lim CJ, Puzon-McLaughlin W, Shattil SJ, Ginsberg MH. Riam activates integrins by linking talin to ras gtpase membranetargeting sequences. J Biol Chem, 2009, 284: 5119-5127

20 Han JW, Lim CJ, Watanabe N, Soriani A, Ratnikov B, Calderwood DA, Puzon-McLaughlin W, Lafuente EM, Boussiotis VA, Shattil SJ, Ginsberg MH. Reconstructing and deconstructing agonist-induced activation of integrin alpha iib beta 3. Curr Biol, 2006, 16: 1796-1806

21 Yang J, Zhu L, Zhang H, Hirbawi J, Fukuda K, Dwivedi P, Liu J, Byzova T, Plow EF, Wu J, Qin J. Conformational activation of talin by riam triggers integrin-mediated cell adhesion. Nat Commun, 2014, 5: 5880

22 Barsukov IL, Prescot A, Bate N, Patel B, Floyd DN, Bhanji N, Bagshaw CR, Letinic K, Di Paolo G, De Camilli P, Roberts GCK, Critchley DR. Phosphatidylinositol phosphate kinase type 1 gamma and beta(1)-integrin cytoplasmic domain bind to the same region in the talin ferm domain. J Biol Chem, 2003, 278: 31202-31209

23 Burridge K, Connell L. A new protein of adhesion plaques and ruffling membranes. J Cell Biol, 1983, 97: 359-367
24 del Rio A, Perez-Jimenez R, Liu R, Roca-Cusachs P, Fernandez JM, Sheetz MP. Stretching single talin rod molecules activates vinculin binding. Science, 2009, 323: 638-641

25 Grashoff C, Hoffman BD, Brenner MD, Zhou RB, Parsons M, Yang MT, McLean MA, Sligar SG, Chen CS, Ha T, Schwartz MA. Measuring mechanical tension across vinculin reveals regulation of focal adhesion dynamics. Nature, 2010, 466: 263-266

26 Roca-Cusachs P, del Rio A, Puklin-Faucher E, Gauthier NC, Biais N, Sheetz MP. Integrin-dependent force transmission to the extracellular matrix by alpha-actinin triggers adhesion maturation. Proc Natl Acad Sci USA, 2013, 110: E1361-E1370

27 Wang XF, Ha T. Defining single molecular forces required to activate integrin and notch signaling. Science, 2013, 340: 991-994

28 Deng QP, Huo YQ, Luo JC. Endothelial mechanosensors: the gatekeepers of vascular homeostasis and adaptation under mechanical stress. Sci China Life Sci, 2014, 57: 755-762

29 Kong F, García AJ, Mould AP, Humphries MJ, Zhu C. Demonstration of catch bonds between an integrin and its ligand. J Cell Biol, 2009, 185: 1275-1284

30 Ebner A, Hinterdorfer P, Gruber HJ. Comparison of different aminofunctionalization strategies for attachment of single antibodies to afm cantilevers. Ultramicroscopy, 2007, 107: 922-927

31 Sarangapani KK, Marshall BT, McEver RP, Zhu C. Molecular stiffness of selectins. J Biol Chem, 2011, 286: 9567-9576

32 Kong F, Li ZH, Parks WM, Dumbauld DW, Garcia AJ, Mould AP, Humphries MJ, Zhu C. Cyclic mechanical reinforcement of integrinligand interactions. Mol Cell, 2013, 49: 1060-1068

33 Zhang XH, Wojcikiewicz E, Moy VT. Force spectroscopy of the leukocyte function-associated antigen-1/intercellular adhesion molecule-1 interaction. Biophys J, 2002, 83: 2270-2279

34 Klein DCG, Ovrebo KM, Latz E, Espevik T, Stokke BT. Direct measurement of the interaction force between immunostimulatory cpg-DNA and tlr9 fusion protein. J Mol Recognit, 2012, 25: 74-81

35 Yan C, Yersin A, Afrin R, Sekiguchi H, Ikai A. Single molecular dynamic interactions between glycophorin a and lectin as probed by atomic force microscopy. Biophys Chem, 2009, 144: 72-77

36 Evans E. Probing the relation between force-lifetime-and chemistry in single molecular bonds. Ann Rev Biophys Biomol Struct, 2001, 30: $105-128$

37 Chen W, Lou J, Zhu C. Forcing switch from short- to intermediateand long-lived states of the alphaa domain generates LFA-1/ICAM-1 catch bonds. J Biol Chem, 2010, 285: 35967-35978

38 Bell GI. Models for the specific adhesion of cells to cells. Science, 1978, 200: 618-627

39 Phillips JC, Braun R, Wang W, Gumbart J, Tajkhorshid E, Villa E, Chipot C, Skee RD, Kalé L, Schulten K. Scalable molecular dynamics with namd. J Comput Chem, 2005, 26: 1781-1802

40 MacKerell A, Jr., Bashford D, Bellott M, Dunbrack RLJ, Evanseck J, Field MJ, Fischer S, Gao J, Guo H, Ha S, Joseph D, Kuchnir L, Kuczera K, Lau FTK, Mattos C, Michnick S, Ngo T, Nguyen DT, Prodhom B, Reiher IWE, Roux B, Schlenkrich M, Smith J, Stote R, Straub J, Watanabe M, Wiorkiewicz-Kuczera J, Yin D, Karplus M. All-atom empirical potential for molecular modeling and dynamics studies of proteins. J Phys Chem B, 1998, 102: 3586-3616

41 Humphrey W, Dalke A, Schulten K. VMD: visual molecular dynamics. J Mol Graphics, 1996, 14: 33-38

42 Lee CK, Wang YM, Huang LS, Lin SM. Atomic force microscopy: determination of unbinding force, off rate and energy barrier for protein-ligand interaction. Micron, 2007, 38: 446-461

43 Evans E, Ritchie K. Dynamic strength of molecular adhesion bonds. Biophys J, 1997, 72: 1541-1555

44 Merkel R, Nassoy P, Leung A, Ritchie K, Evans E. Energy landscapes of receptor-ligand bonds explored with dynamic force spectroscopy. Nature, 1999, 397: 50-53

45 Marshall BT, Long M, Piper JW, Yago T, McEver RP, Zhu C. Direct observation of catch bonds involving cell-adhesion molecules. Nature, 2003, 423: 190-193

46 Chen W, Lou J, Evans EA, Zhu C. Observing force-regulated conformational changes and ligand dissociation from a single integrin 
on cells. J Cell Biol, 2012, 199: 497-512

47 Yago T, Lou J, Wu T, Yang J, Miner JJ, Coburn L, Lopez JA, Cruz MA, Dong JF, McIntire LV, McEver RP, Zhu C. Platelet glycoprotein ibalpha forms catch bonds with human wt vwf but not with type $2 b$ von willebrand disease vwf. J Clin Invest, 2008, 118: 3195-3207

48 Lee CY, Lou J, Wen KK, McKane M, Eskin SG, Ono S, Chien S, Rubenstein PA, Zhu C, McIntire LV. Actin depolymerization under force is governed by lysine 113:glutamic acid 195-mediated catchslip bonds. Proc Natl Acad Sci USA, 2013, 110: 5022-5027

49 Guo B, Guilford WH. Mechanics of actomyosin bonds in different nucleotide states are tuned to muscle contraction. Proc Natl Acad Sci USA, 2006, 103: 9844-9849

50 Michael Feig, John Karanicolas, III CLB. Mmtsb tool set: enhanced sampling and multiscale modeling methods for applications in structural biology. J Mol Graphics Model, 2004, 22: 377-395

Open Access This article is distributed under the terms of the Creative Commons Attribution License which permits any use, distribution, and reproduction in any medium, provided the original author(s) and source are credited.

\section{Supporting Information}

Figure S1 Free cysteine residues in talin F2F3/RS complex.

Figure S2 The adhesion frequency between F2F3 functionalized AFM tips and the RS domain coated on the petri dishes.

Figure S3 Alignment of three different structures of talin F3/RS complex after fitting RS domain: Crystal structure (grey), Mode 1 (green) and Mode 2 (purple).

Figure S4 The decay of survival probability between talin F2F3 and RS under different forces.

Table S1 The goodness of fitting with one-, two-state models

The supporting information is available online at life.scichina.com and link.springer.com. The supporting materials are published as submitted, without typesetting or editing. The responsibility for scientific accuracy and content remains entirely with the authors. 